\title{
Skin Tuberculosis: A Case Report
}

\author{
S AHMAD ${ }^{\mathrm{a}}$, I FARUK ${ }^{\mathrm{b}}$, MM HUSSAIN $^{\mathrm{c}}$, MA FAIZ ${ }^{\mathrm{d}}$
}

Summary:

A 17 year old boy presented with multiple non healing ulcers over the right lower limb and left upper limb for 13 years which were non responsive to varieties of treatment modalities used in his locality. There were also contracture deformities of the affected limbs. Clinically the diagnosis was quite confusing. but histopathology of

\section{Introduction:}

Tuberculosis of the skin is caused by M. tuberculosis , M. bovis and under certain conditions the bacillus Calmettee- Guerin (BCG), an attenuated strain of $\mathrm{M}$. bovis. ${ }^{1}$ With respect to Mycobacterial skin disease , the so called atypical mycobacteria may be the cause of skin disease more frequently than $M$. tuberculosis. ${ }^{2}$ Cutaneous tuberculosis (TB) is essentially an invasion of the skin by Mycobacterium tuberculosis, the same bacteria that causes pulmonary TB. ${ }^{3}$ Cutaneous TB is a relatively uncommon form of extrapulmonary TB. Even in countries such as India and China where TB still commonly occurs, cutaneous TB cases are $\operatorname{rare}(<0.1 \%)^{2}$. Direct infection of the skin or mucous membranes from an outside source of mycobacteria results in a initial lesion called the tuberculous chancre. The chancres are firm shallow ulcers with a granular base. They appear about 2-4 weeks after mycobacteria enter through broken skin. The immune response of the

a. Dr. Sami Ahmad, MBBS, FCPS (Surgery), Junior Consultant, Surgery, Nowabgonj Health Complex, Dhaka.

b. Dr. Imtiaz Faruk, MBBS, FCPS (Surgery), Rs (General), Dhaka Medical College Hospital, Dhaka.

c. Professor Md. Margub Hussain, MBBS, FCPS (Surgery), FICS, MHPED (UNSW, Australia), Professor of Surgery, Dhaka Medical College And Hospital, Dhaka.

d. Professor M A Faiz, MBBS, FCPS ( Medicine), FRCP (Edin), PhD ( Uk), Director General, Health Services, Mohakhali, Dhaka.

Address of Correspondence: Dr, Sami Ahmad, MBBS, FCPS (Surgery), Junior Consultant, Surgery, Nowabgonj Health Complex, Dhaka, E-mail: dr.sami39@yahoo.com.

Received: 12 September, 2007

Accepted: 23 March, 2009 specimen from skin lesion confirmed it as a case of skin tuberculosis. The patient was put on anti TB drugs and the ulcers responded well and started to heal rapidly. Some reconstructive surgery will be done for his contractures after completion of the anti $\mathrm{TB}$ drug regimen and proper healing of ulcers.

(J Bangladesh Coll Phys Surg 2009; 27: 107-111)

patient and the virulence of the mycobacteia determine the type and severity of the cutaneous TB.The types are TB verrucosa cutis, Lupus vulgaris,

Scrofuloderma, Miliary TB and Tuberculid TB. The diagnosis is usually made or confirmed by a skin biopsy. Typical tubercles are caseating epithelioid granulomas that contain acid-fast bacilli. Other tests that may be necessary include: Tuberculin skin test(Mantoux), sputum culture (It may take a month or longer for results to be reported), Chest X-ray and other radiological tests for extrapulmonary infections.Patients with pulmonary or extrapulmonary TB need to be treated with antitubercular drugs. This usually a combination of antibiotics( Isoniazid, Rifampicin, Pyrazinamide and

Ethambutol ) given over a period of 6 months. ${ }^{4}$ Involvement of other organs along with skin may need treatment for several months and sometimes years. Patients with TB infection but no active disease must also be treated with antitubercular drugs to prevent development of active disease. Occasionally surgical excision of localized cutaneous TB is recommended.

\section{Case Report:}

The patient was a 17 year old boy from Norshingdi, got admitted in surgery unit II of Dhaka Medical College Hospital (DMCH) on $12^{\text {th }}$ March, 2007 with the complaints of multiple ulceration over the right lower limb for last 13 years, left upper limb for 8-9 years and progressive stiffening of the joints of affected limbs for the last one year.Initially there was a very small nodule on the sole of the right foot wich ulcerated and gradually spread over the whole lower 
limb. At the age of 7 years after getting a cut injury over the base of left thumb same scenario began.These ulcers were non-responsive to different medications. It was associated with variable episodes of fever, loss of appetite and weight loss. During the whole period of illness he never had any history of cough and haemoptysis, significant bowel and bladder complaints or any other systemic problems.

$\mathrm{He}$ is also not a known diabetic patient. He gave no history of childhood skin disease. He received some vaccines but the exact nature was not clear. He also gave no history of contact with TB patient. On examination the patient's body build and nutritional status was found to be below average. $\mathrm{He}$ was anaemic and oedema was present on the affected leg with no significant lymphadenopathy. His right lower limb showed multiple painful ulcers of variable size with discharging pus, floors were covered with slough and bleeded after removing the slough. There were also some healing ulcers which had scabs. Same features were seen on the left upper limb but with more crust and slough formation. No nerve thickening was found. The skin was thickened, hyperkeratotic. All other systemic examinations revealed no abnormalities and testes were normal. On investigations, total WBC count was $9800 / \mathrm{cu} \mathrm{mm}$, differential count were neutrophil $66 \%$, lymphocyte $31 \%$, eosinophil $2 \%$, monocyte $1 \%$, Haemoglobin was $65 \%$, ESR $70 \mathrm{~mm}$ in $1^{\text {st }}$ hour, blood sugar 2 hours after breakfast was $4.8 \mathrm{mmol} / 1$, serum total protein 88 $\mathrm{gm} / 1$, serum albumin $34 \mathrm{gm} / 1$, serum globulin 54 $\mathrm{gm} / \mathrm{l}$, serum A:G was $0.6: 1$, serum creatinine 1.4 $\mathrm{mg} / \mathrm{dl}$, MT test was positive (30 $\mathrm{mm}$ in diameter), HIV screening test was negative. Chest X-ray, X-rays of the affected limbs, Ultrasonogram of whole abdomen were normal. Skin scrapping for fungal infection was also negative. Skin biopsy was taken and histopathology report revealed that this was a case of skin tuberculosis. Fig-1. After confirming the diagnosis antitubercular drug was started in the form of isoniazid, rifampicin, pyrazinamide and ethumbutol for the initial 2 months and isoniazid and rifampicin for next 4 months $^{4}$. During that period the wound was closed regularly but kept it open. The patient is on his third month of treatment now and the ulcers have already healed a lot. Fig-2, Fig-3, Fig-4, Fig-5. After completion of this antitubercular drug

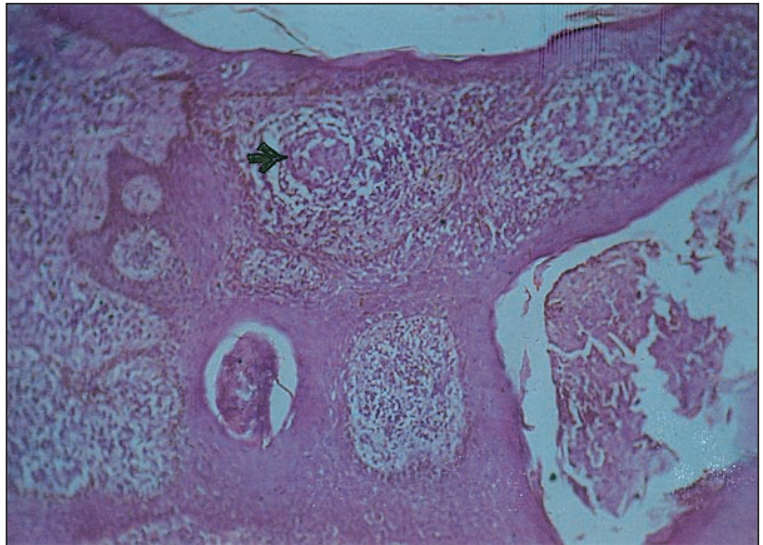

Fig.-1: Photomicrograph of skin biopsy showing features suggestive of tuberculosis. Black arrow indicate granuloma.

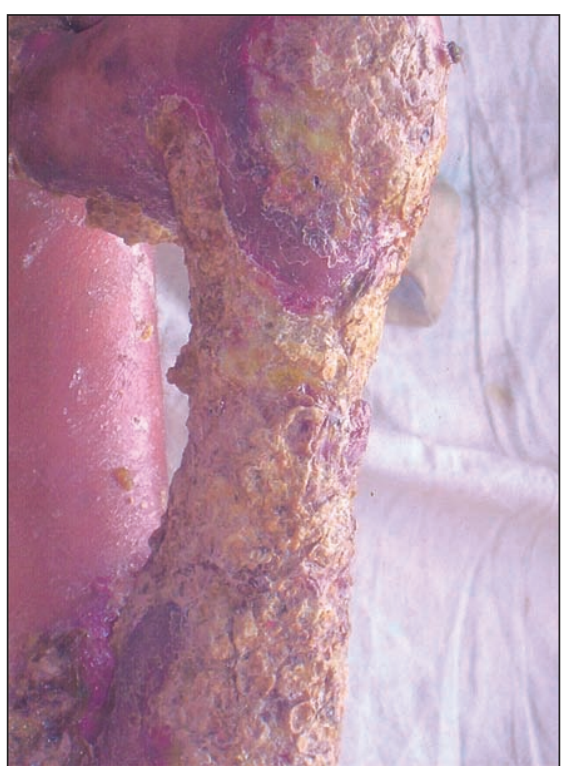

Fig.-2: Lesion of the hand before starting anti $T B$ drugs.

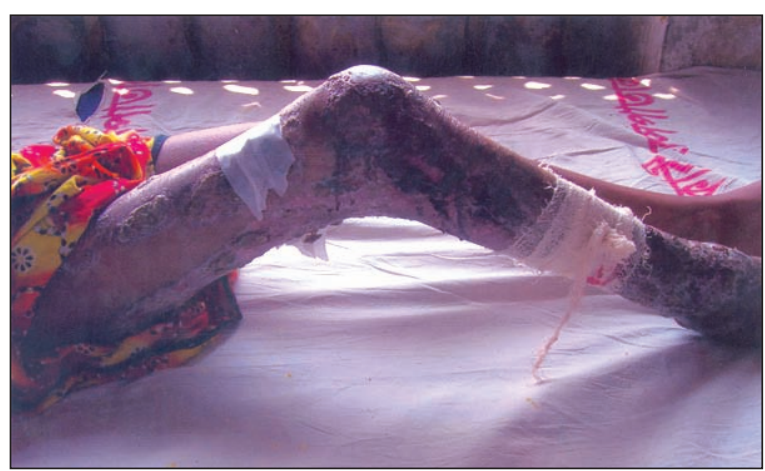

Fig.-3: Lesion of the foot before treatment with anti TB drugs. 


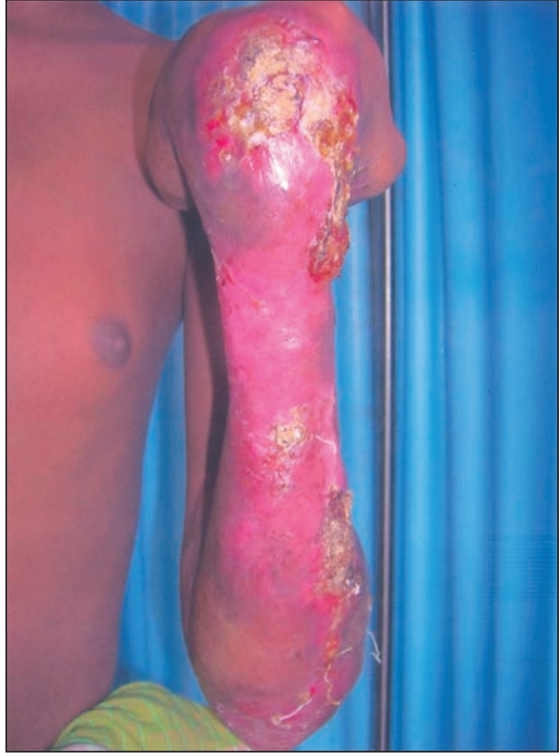

Fig.-4: Lesion of the hand after 3 months treatment with anti TB drugs.

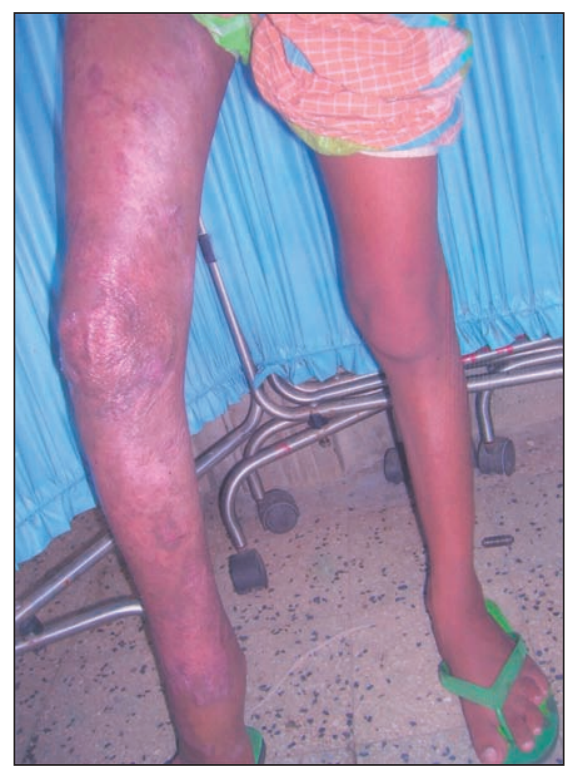

Fig.-5: Lesion of the foot after 3 month treatment with anti TB drugs.

regimen this patient will receive some form of reconstructive surgery to relieve his wound contracture.

\section{Discussion:}

Cutaneous tuberculosis is caused by Mycobacterium Tuberculosis and a member of a group of closely related organisms in the $M$. tuberculosis complex: Mycobacterium africanum, Mycobacterium bovis, Mycobacterium microti, and M. tuberculosis are also responsible. World incidence of TB increased with population density and urban development. During industrial revolution in Europe (1750), it was responsible for more than $25 \%$ of adult deaths. Indeed, in the early $20^{\text {th }}$ century, TB was the leading cause of death in the United States ${ }^{3}$. Although 1 of 3 individuals globally is infected with tubercle bacillus, the incidence of cutaneous TB appears to be low. In areas such as India or China where TB prevalence is high, cutaneous manifestations of TB (overt infection or tuberculids ) are found is less than $0.1 \%$ of individuals seen in dermatology clinics.In a 10 year (1983-1992) retrospective survey of patients seen in government dermatology clinics in Hong Kong, the detected Incidence of cutaneous TB among patients was 179 per $267,089(0.07 \%)$. Among patients with cutaneous TB, 15\% had classic cutaneous TB (approximately $5 \%$ each of lupus vulgaris, TB verrucosa cutis and scrofuloderma.) and $85 \%$ had tuberculids. In a tertiary care hospital in northern India, $0.1 \%$ of dermatology patients seen from 1975 1995 had cutaneous TB. Lupus vulgaris was the most frequent manifestation (55\%), followed by scrofuloderma (27\%), TB verrucosa cutis $(6 \%)$, tuberculous gumma (5\%) and tuberculids (7\%). The incidence of patients with cutaneous TB seen from 1980-1993 in a hospital dermatology clinic in Madrid was $16(0.14 \%)$ in

10,304. 160 cases of tuberculosis of the skin seen in the Government Dermatological Clinics in Hong Kong 5 are reviewed. Tuberculosis verrucosa was the commonest form and account for $46 \%$ of cases. The onset was in below the age of 20 in $77 \%$ of these patients. In a study of cutaneous tuberculosis took place in Morocco ${ }^{6}$ two hundred and sixteen cases of cutaneous tuberculosis were identified and included. Men and women were equally affected. The mean patient age was 29 years. Major clinical types of cutaneous tuberculosis were scrofuloderma and gumma $(72 \%)$, lupus vulgaris $(12 \%)$, tuberculosis verrucosa cutis $(7 \%)$, tuberculids $(6 \%)$, orificial tuberculosis $(1 \%)$ and tuberculous chancre $(1 \%)$. Systemic involvement was seen in $35 \%$ of cases. Where performed $(66 \%), 81 \%$ of subjects had 
positive Mantoux skin test. Lesion biopsy for histopathologic study was performed in $81 \%$ of patients and showed classical tuberculous findings in $57 \%$. Mycobacterium tuberculosis was isolated in culture from $9 \%$ of patients.

Primary inoculation TB results from direct introduction of mycobacteria into the skin or mucosa of an individual who was not previously infected with TB or was immunized with the $M$ bovis strain Bacilli Calmette Guerin (BCG). Since mycobacterias do not penetrate intact skin, initiation of infection almost always follows an injury, usually in children. Common sites include the face and other exposed skin $^{3}$. This presentation is quite similar to our case. But there are also some variations in development of skin tuberculosis such as a case published in a Turkey Journal. ${ }^{6}$ where the authors reported a case of Tuberculosis verrucosa cutis in a patient with long standing generalized lichen planus of more than 20 years history and improvement of lichen after antitubercular polychemotherapy. The authors here tried to reflect a possible reciprocal causal relationship between two cutaneous conditions of different natures. In another case published in the American Journal of Nephrology ${ }^{8}$ skin tuberculosis with atypical mycobacteria 8 years after combined pancreas- kidney transplantation where they report on a Mycobacterium marinum infection in a diabetic woman 8 years after undergoing a combined pancreas-kidney transplantation. This is, to their knowledge, the first case report on an isolated skin infection with atypical mycobacteria after simultaneous pancreas- kidney transplantation. A genetic probe categorization revealed an infection with $M$. marinum. Skin tuberculosis caused by $M$ marinum is an uncommon complication in kidney or pancreas-kidney transplant recipients, hence the diagnosis can be delayed TB is an airborne communicable disease that occurs after inhalation of infectious droplets expelled from patients with laryngeal or pulmonary TB during coughing, sneezing or speaking. Each cough can generate more than 3000 infectious droplets. Droplets are so small (1-5 micro meter) that they remain airborne for hours. The probability that disease transmission will occur depends upon the infectiousness of the tuberculous patient, the environment in which the exposure takes place, and the duration of exposure ${ }^{3}$. But this is not always the same in skin tuberculosis. A rare case published in the American Journal of Tropical Medicine and Hygiene in 2004 reported a case of tuberculosis transmission from a patient with skin lesion and a negative sputum smear ${ }^{9}$. Here the author mentioned about a five-month- old girl who presented with miliary tuberculosis and multiple intracranial tuberculomas and her disease was actually transmitted from a 20 year old man presented with skin tuberculosis. M. tuberculosis isolates from the man and the infant matched exactly on both IS6110 restriction fragment length polymorphism analysis and spacer oligonucleotice typing. It has been estimated that patients with negative acid- fast smears cause 17 percent of new infections and present serious challenges to tuberculosis control. The common investigations that can be done for cases of skin tuberculosis are Chest $\mathrm{X}$ - ray, Tuberculin test( Mantoux), sputum smear and staining for acid fast bacilli, skin biopsy and histopathological examinations 1 were all done in this case and the results were also quite similar to other cases of skin tuberculosis. In presented case treatment was started with initial four drugs regimen comprising of isoniazid, rifampicin, ethumbutol, and pyrazinamide for two months and isoniazid and rifampicin for next 4 months. Though there is some mentioning about role of surgery in case of treating skin tuberculosis but this still

controversial and in presented case any surgery was not done for the wound. But the patient will receive some reconstructive surgery to relieve his wound contracture on a later period.

\section{Conclusion:}

Any longstanding ulcer not responding to conventional treatment needs assessment and treatment in expert hands before implicating their mutilating effects on human body.

\section{References:}

1. Tappeinar G, Wolff K. Tuberculosis and other Mycobacterial Infection. In Freedberg IM. Eisen AZ. Wolff K. Austen KF. et al (editors) Fitzpatrick's Dermatology in General Medicine , 6th edition , Newyork, McGRAW- HILL, 2003; P:1933-1949.

2. Dalovisio JR, Pankey GA. Dermatologic manifestation of nontuberculous mycobadterial disease, Infect. Dis. Clin. North Am 8 : 677, 1994. 
3. Meltzer MS, Nancy CA,Verma S, Vinson RP, Meffert J. Cutaneous tuberculosis the article; e Medicine; November $20,2006$.

4. American Thoracic Society: Treatment of tuberculosis and tuberculous infection in adults and children. Am J Respir. Crit. Med. 140: 1359, 1994.

5. Wong KO, Lee KP, Chiu SF (1968). Tuberculosis of the skin in Hong Kong (a review of 160 cases), British Journal Of Dermatology 80 (7): 424-429.

6. Zouhair K, Akhdari N, Nejjam N, Ouazzani T. Lakhdar H, Dermatoloty Department.USC Ibn Rochd, Cassablanca. Morocco. Cutaneous Tuberculosis in Morocco.
7. Erbagei Z, Tuncel A. Bayram N. Erkilic S. Bayram A. Tuberculosis verrucosa cutis in a patient with long standing generalized lichen planus, Improvement of lichen after antitubercular polychemotherapy, Journal of Dermotological Treatment, Volume 17, Issue 5 September, 2006. 314- 318.

8. Schmekal B, Janko O, Zazgonik J, Schinko H, Bogner S, Syre G, Biesenbach G. Skin tuberculosis with atypical Mycobacteria 8 years after combined Pancreas- Kidney Transplantation. American Journal of Nephrology, 2002, 22, 566-568.

9. Riehl G, Kopf O, Strakosch EA. Am.J. Trop. Hyg. Volume350: 2527-2528 June 10, 2004. Number 24. 